\title{
Decision Tree Based Uncertainty Framework for Water Accounting
}

\author{
$\underline{\text { Jin } H^{1}}$, Barry S. ${ }^{1}$, Paydar $Z^{2}{ }^{2}$, Shao $Q^{3}$ and van Dijk $A^{2}$ \\ ${ }^{1}$ CSIRO Mathematics, Informatics and Statistics, GPO Box 664, Canberra ACT 2601, Australia. Email: \\ Warren.Jin@csiro.au. \\ ${ }^{2}$ CSIRO Land and Water, GPO Box 1666, Canberra 2601, Australia \\ ${ }^{3}$ CSIRO Mathematics, Informatics and Statistics, Private Bag 5, PO Wembley, WA 6913, Australia.
}

\begin{abstract}
Water accounting is becoming a common term in water resources management, especially in Australia after the Council of Australian Governments (COAG) reached an intergovernmental agreement on the National Water Initiative (NWI) in 2004. Water accounting aims to provide a complete picture of water resources for a region over a reporting period. It provides quantitative information about all water resource components such as inflows, outflows, rainfall, evaporation, water use, surface water availability and change, groundwater availability and change etc on a consistent basis. It has already been occasionally used in water resources management and planning processes. For example, the Commonwealth Water Act 2007 assigns the Bureau of Meteorology (the Bureau hereinafter) the role of "compiling and maintaining water accounts for Australia, including a set of water accounts to be known as the National Water Account." Among other obligations under the Act, the Bureau is required to publish this National Water Account (NWA) annually in a form readily accessible by the public. The NWA will provide reliable information such as water rights, water availability and water use that has previously been difficult to access or unavailable to general users in a standardised form. The Bureau, together with water agencies and other government agencies, has successfully prepared and published Pilot NWA for 2007-2008, and will publish the first NWA in 2011.
\end{abstract}

Uncertainty of a measurand is "a parameter associated with the result of a measurement or estimation that characterises the dispersion of the values that could reasonably be attributed to the measurand." Most, if not all, line items in water accounting are subject to uncertainties. The sources of uncertainty vary, depending on the origin of data and processing procedures. Possible uncertainty sources include direct measurement uncertainty, indirect measurement uncertainty (processing errors from recording, estimation, interpolation, aggregation, sampling, etc), and hydrological model uncertainty (model structure and model parameters). Quantitative uncertainty information in water accounting can encourage users to query the precision of the underlying data and information, thereby improving their understanding of the nature of water stocks and flows. It can help identify the main contributors to the uncertainty thus enabling resources to be allocated appropriately in order to improve the accounting in future. It can also improve risk control in informed water management and planning. Thus, it is crucial to include uncertainty in water accounting, such as the NWA.

As an activity under the Water Resources Information Research and Development Alliance (WIRADA) between the Bureau and CSIRO, we develop a consistent uncertainty framework for assessing, quantifying and reporting of quantitative uncertainty for water accounting. The proposed hierarchical decision-tree-based uncertainty framework is intended to help systematically choose and apply consistent and reliable approaches for quantifying and reporting uncertainty information about water accounting items. For each line item, the decision tree considers its nature, source and logical foundation. It will provide a pathway to some end point through a couple of simple decisions. For each end point (leaf node) in the decision-tree, we recommend a practical uncertainty analysis technique, ranging from classical uncertainty analysis techniques, uncertainty propagation law, Monte Carlo, literature to expert elicitation. Hierarchical structures are ubiquitous in the water accounting. They may originate from geographical hierarchy of reporting entities like subcatchment, catchment, and a nation, or from reporting detail hierarchy like detailed, intermediate, and simple water accounts, or from temporal hierarchy like monthly and yearly water accounts. We also suggest techniques to aggregate uncertainty from a lower level of accounting items. We simply list software implementation possibilities, including add-ins for spreadsheet like Microsoft Excel, standalone software, and general statistical software. For the work in progress, we also discuss a couple of issues in this preliminary uncertainty framework for water accounting.

Keywords: Water accounting; quantitative uncertainty estimation; hydrological modelling; error propagation; Monte Carlo; expert elicitation 


\section{INTRODUCTION}

Water accounting is becoming a common term in water resources management, especially in Australia after the Council of Australian Governments (COAG) reached an intergovernmental agreement on the National Water Initiative (NWI) in 2004 [Shao et al., 2009]. Water accounting aims to provide a complete picture of water resources for a region over a given reporting period with reliable quantitative information about all water resource components on a consistent basis. It has already been occasionally used in water resources management and planning processes, such as describing the status of water resources, protecting fish, and highlighting the drivers, impacts and responses of water management policies and practices, see [Jin et al., 2011] and references therein.

Through the Commonwealth Water Act (2007), the Bureau of Meteorology (the Bureau hereainfter) has statutory responsibility for compiling and delivering comprehensive water information across Australia, known as the National Water Account (NWA). It is defined as "a systematic process of identifying, recognising, quantifying, reporting, and assuring information about water, the rights and other claims to that water, and the obligations against that water" [Bureau of Meteorology, 2010]. The NWA will provide a valuable window into the management of Australia's water resources at the national and regional scale. It will support the important NWI and will disclose the total water resource, the volume of water available for abstraction, the rights to abstract water, and the actual abstraction of water for economic, social, cultural and environmental benefit across Australia. The NWA will be produced for a financial year from 1 July to 30 June. The Bureau has been proposing to use the best available data [Barratt et al., 2009], produce and review via standardised processes [Barratt and Nation, 2009; Frost et al., 2009], such as Methods Pilot [Bureau of Meteorology, 2010], keep consistent with Australian Water Accounting Standards, say [Water Accounting Standards Board, 2010]. The Bureau has published the Pilot NWA [Bureau of Meteorology, 2010], and will publish the first NWA in 2011.

To make the NWA a significant contribution towards the improved management of Australia's water resources, the Bureau is further developing methods for water accounting standards, techniques and procedures. One required development is to include uncertainty information in water accounting. All reported items in a physical water account, i.e., components in the physical water system, are prone to uncertainty due to measurement, modelling or subjective judgment uncertainties [Frost et al., 2009; Lowe et al., 2006]. For example, hydrologic models are subject to input data uncertainty, structural uncertainty, model parameter uncertainty, temporal uncertainty [Beven and Binley, 1992; Gupta et al., 2005; Kuczera et al., 2006]. Quantification of these uncertainties provides transparency and robustness in the accounting methodology, and enables stakeholders to incorporate this information in an appropriate way in decision making to optimise the social, economical and environmental outcomes of water resource management. Thus, it is crucial to include uncertainty information in water accounts such as the NWA [Jin et al., 2011; Lowe et al., 2006; Lowe et al., 2009; The Bureau of Meteorology, 2010].

Where possible, we suggest a quantitative uncertainty expression for reporting, which is widely used in literature and also recommended by Intergovernmental Panel on Climate Change for national greenhouse gas inventories [Penman et al., 2000], as follows “Where there is sufficient information to define the underlying probability distribution for conventional statistical analysis, a 95 per cent confidence interval should be calculated as a definition of the range." It indicates that, for the estimated quantity, the confidence interval is specified by the confidence limits defined by the interval between the 2.5 percentile and 97.5 percentile of its cumulative distribution function. For example, the uncertainty for the 2924ML of groundwater extractions (Figure 1) is [2485ML, 3743ML] [Jin et al., 2011].

Together, the Bureau and CSIRO have established the Water Information Research and Development Alliance (WIRADA). WIRADA is a strategic investment of $\$ 50 \mathrm{~m}$ over five years that will yield most of the innovation required by the Bureau to fulfil its national water information mandate. As an activity under the WIRADA, we are

Beta probability function for Groundwater extractions

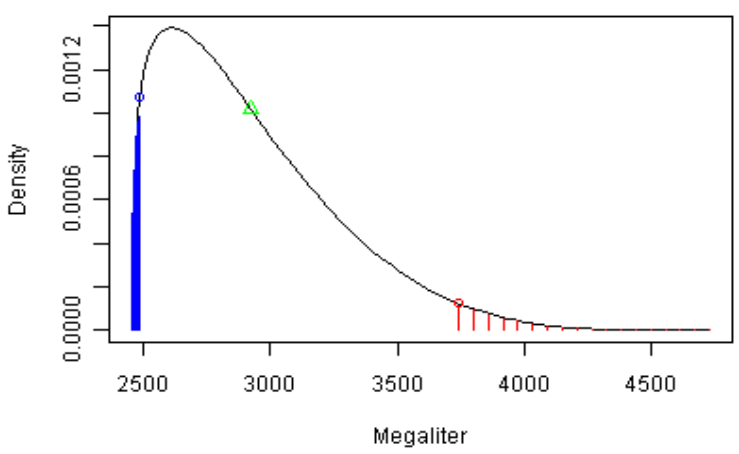

Figure 1. Beta distribution for groundwater extractions in the water account 2005/2006 for the Werribee River catchment, Victoria. The uncertainty lower bound, the mean, and the uncertainty upper bound are indicated. 
developing a consistent framework for assessing, quantifying and reporting of uncertainty for water accounting. The uncertainty framework aims to help the process of choosing and applying consistent and reliable approaches for quantifying and reporting uncertainty information about water accounting entities, in a systematic way. Our proposed hierarchical decision-based uncertainty framework consists of two parts: (a) a decision tree to handle each possible line item in water accounts (Section 2), and (b) some methods to address hierarchical structure common in water accounting (Section 3). After introduction of the uncertainty framework, we describe possible implementation in Section 4, followed by conclusion and discussions in Section 5.

\section{DECISION-TREE BASED UNCERTAINTY ANALYSIS FOR INDIVIDUAL TERMS}

We first define an individual term as a term whose quantity comes from an independent data source, instead of aggregation of some terms at a subordinate level. So, an individual term could be one line item in a detailed water account, or one term in an intermediate water account if the detailed water account is unavailable in such as the Pilot NWA [Bureau of Meteorology, 2010].

In general, based on the information available for uncertainty quantification, we have a list of categories of uncertainties. Roughly going from simple to more complicated techniques, we give a decision-tree where for each category, a pathway is provided to some end point through a couple of decision nodes (diamond nodes in Figure 2) considering its nature, source and logical foundation. For each end point (rectangle node) in the decision-tree, we recommend a practical and appropriate uncertainty analysis technique. We discuss these nodes with possible examples in the following subsections, starting from the top in Figure 2.

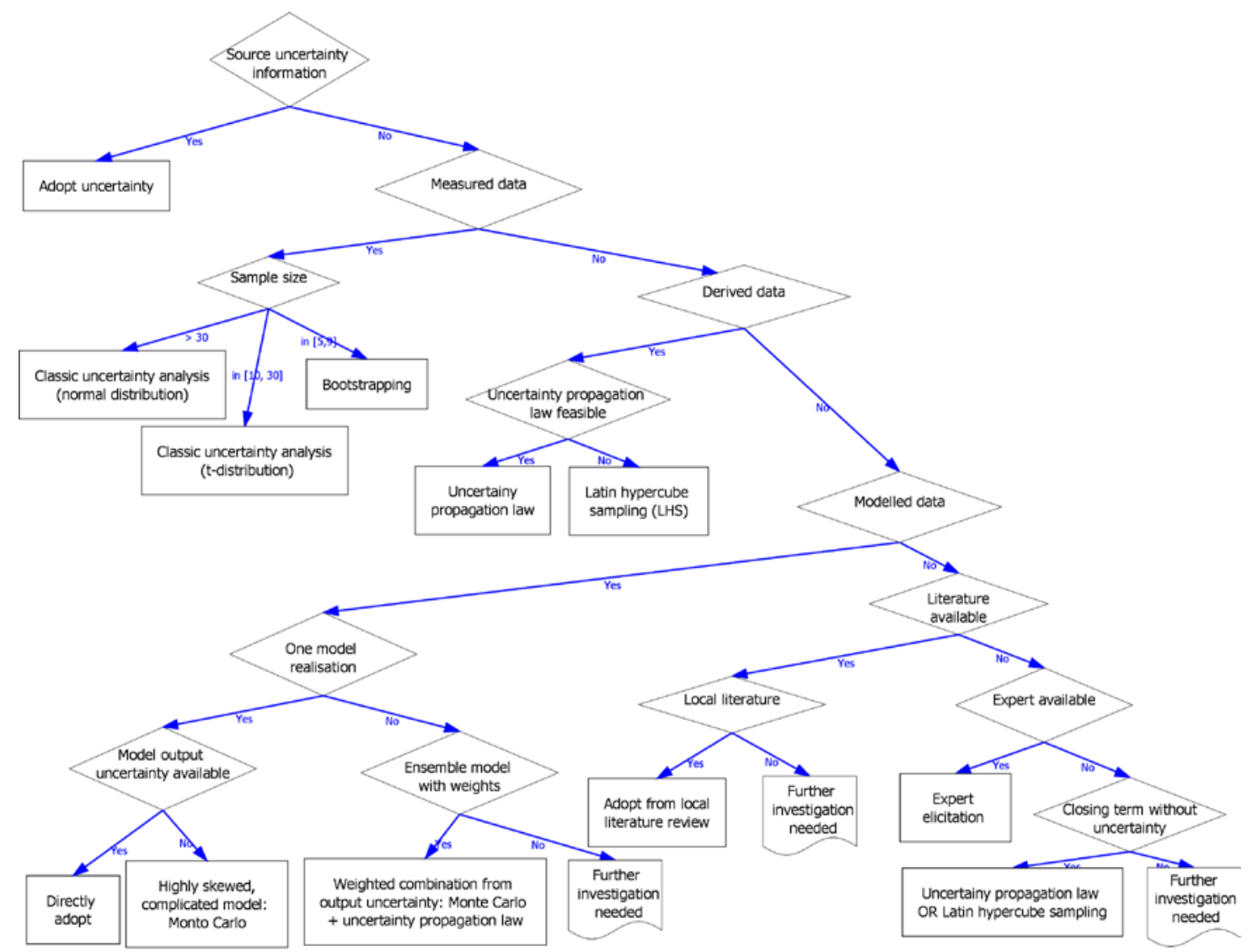

Figure 2. Decision-tree structure for quantifying uncertainty of individual terms in water accounts

\subsection{Source uncertainty information}

Source uncertainty information means that some uncertainty quantification is credibly available for a water term. For example, for the precipitation terms the uncertainty in the interpolated daily rainfall surface is currently being supplied operationally by the Bureau's AWAP (the Australian Water Availability Project) [Frost et al., 2009]. Thus, the first decision is to ascertain whether there is source uncertainty information for an individual water term. We would simply adopt the uncertainty quantification if it is at the given confidence level. 


\subsection{Measured data}

Measured data indicates that individual terms are measured directly and repeatedly. In other words, there is a list of measurements available for a single water term, e.g., desalinated water volume from a series of measured data. If these measured data are independent and identically distributed, the uncertainty estimation could be derived.

When there are five or more repeated data measurements, and they are a random representative sample of the quantity of interest, it is possible to apply statistical techniques to quantify uncertainty. When the number of measurements is reasonably large, say, greater than 30, we can use classic uncertainty analysis techniques. If a random variable $x$ has $N$ measurement values $x_{1}, x_{2}, \cdots, x_{N}$ (which are real values) with equal probability, then the standard uncertainty $u_{x}$ is given $u_{x}=\sqrt{\frac{1}{N(N-1)} \sum_{i=1}^{N}\left(x_{i}-\bar{x}\right)^{2}}$ with the mean $\bar{x}=\frac{x_{1}+x_{2}+\cdots+x_{N}}{N}=\frac{1}{N} \sum_{i=1}^{N} x_{i}$.

Under the assumption that the underlying distribution is symmetric, as our purpose of the uncertainty statement is to provide coverage with a high level of confidence $\alpha$, say 95\%, an expanded uncertainty is obtained by multiplying the standard uncertainty by a coverage factor $k$ as:

$$
U_{x}=k u_{x},
$$

The coverage factor $k$ is chosen to be the $(\alpha+1) / 2$ quantile value from some distribution table at the given confidence level. When the number of measurements is reasonably large, say $>30$, we use normal distribution, i.e., $k=1.96$ for $\alpha=95 \%$. When it is between 10 and 30 , we use $t$-distribution with $N-1$ degrees of freedom [Penman et al., 2000], Then $\left[\bar{x}-U_{x}, \bar{x}+U_{x}\right.$ ] gives the confidence interval, or uncertainty, at the confidence level of $\alpha$.

When there are only a few measurements, but normally larger than 4 , bootstrapping is a suitable choice. The premise of bootstrapping uncertainty analysis is fairly straightforward. It simulates the population based on the sample available, to derive robust estimates of the statistical properties of a population parameter such as mean and confidence intervals. It constructs samples by sampling with replacement from the original sample, (that is, some elements may occur several times in the constructed samples), and then calculate the population parameter based on the re-sampled data. The bootstrap sampling is repeated a great number of times, say, 1,000 [Penman et al., 2000]. From the sampling distribution of these constructed samples, we can compute a robust approximation to the 'true' population sampling distribution, including the confidence interval [Davison and Hinkley, 1997].

\subsection{Derived data}

Derived data refers to terms that are estimated from other measured data with a simple deterministic function with few (e.g. less than three) uncertain parameters. A water term of interest may consist of two other components that are added, subtracted, multiplied, or divided. For example, annual reservoir evaporation is accumulated from daily pan evaporation adjusted for influence of the bird guard [Lowe et al., 2009]. When processing becomes more complicated, we may better regard them as modelled data, which is discussed in Section 2.4 .

If underlying measured data follow Gaussian distributions and their uncertainties are relatively small, say, the standard deviation divided by the mean value is less than 0.3 , the uncertainty propagation law is recommended [Penman et al., 2000]. It could be formulated as follows. If the relationship between the measurand or a derived variable $y$ and $x_{i}(i=1, \ldots, n)$ is written in a general form as $y=f\left(x_{1}, x_{2}, \cdots x_{n}\right)$. Then the standard uncertainty (or standard deviation) for $y$ could be estimated as follows

$$
u_{y}=\sqrt{\sum_{i=1}^{n}\left(\frac{\partial f}{\partial x_{i}} u_{x_{i}}\right)^{2}+\sum_{i=1}^{n} \sum_{j \neq i}^{n}\left(\frac{\partial f}{\partial x_{i}} \frac{\partial f}{\partial x_{j}} u_{x_{i} x_{j}}{ }^{2}\right)}
$$

where $u_{x_{i} x_{j}}$ is the estimated covariance between $x_{i}$ and $x_{j}$; and $\partial f / \partial x_{i}$ is the partial derivative. Then the coverage factor $k$ in Eq (1) depends on the effective degrees of freedom $v_{\text {eff }}$ of $u_{y}, v_{\text {eff }} v_{\text {eff }}=u_{y}^{4} / \sum_{i=1}^{n}\left(\frac{\partial f}{\partial x_{i}}\right)^{4} \frac{u_{x_{i}}{ }^{4}}{v_{x_{i}}}$ 
where $v_{x_{i}}$ is the degrees of freedom of $u_{x_{i}}$. When $v_{\text {eff }}$ is greater than 30, a Gaussian distribution is preferred to a $t$-distribution to derive $k$.

When uncertainty propagation law is not applicable, we can use stratified sampling, e.g. Latin Hybercube Sampling (LHS). Stratified sampling divides a given input distribution into intervals, and then generates samples from each interval. For efficient sampling (i.e. fewer model runs), intervals are typically constructed so that each has an equal probability of occurrence. In LHS, for more efficient sampling, each sample is the only one in each axis-aligned hyperplane containing it.

\subsection{Modelled data}

Modelled data relates to water terms estimated using more complicated procedures, such as water balance simulation models and rainfall-runoff models, with more than three parameters. The uncertainty of model output, focus of this section, depends heavily on how the model is identified, structured, and calibrated.

There can be one or more model realisations for a water term. A model realisation means a specification of parameter setting, parameters' characteristics, and structure of a specific model. Both different parameter settings and/or different structures may lead to different model realisations [Gupta et al., 2005].

In practice, quite commonly there may be only one single model realisation. In that case we can adopt the model output uncertainty information directly if such information is available for the given confidence level. For most model calibration techniques, like minimising least squares and maximising likelihood [Gupta et al., 2005; Kuczera et al., 2006], there is little uncertainty information available for model output. We recommend Monte Carlo techniques that use statistical sampling techniques to obtain a probabilistic approximation to the solution of an equation or distribution. It can trace out the structure of the distributions of the model output. This distribution, possibly asymmetric, is mapped by calculating the results (realisations) for a large number of random samples drawing from the distribution functions of input data and parameters of the model. Preexisting information about correlations between input variables can be incorporated in the Monte Carlo analysis. It requires the analyst to specify probability distributions of all inputs and parameters, and the correlations between them. Without other knowledge, the model input quantities distributions could be assumed to be Gaussian distributions as a first estimate and then refined. If the bounds of an input quantity are known, we can use truncated Gaussian distributions, uniform distributions or triangle distributions. Guidelines for choosing these distribution can be found in [Penman et al., 2000].

Hydrological systems are open and complex. This often renders them prone to multiple interpretation and mathematical descriptions regardless of the quality and quantity of data available. There is a growing tendency among hydrologists to propose several alternative hydrologic models for a site and use various criteria to rank these models, or weight and average predictions and statistics generated by multiple models. This can generate a set of model realisations. If an individual term can be regarded as a weighted average of output from a set of model realisations, such as from GLUE (Generalized Likelihood Uncertainty Estimation) [Beven and Binley, 1992], we could quantify output uncertainty for each and every model realisation based on Monte Carlo simulation as we discussed above. After that, using uncertainty propagation law (Section 2.3), we can obtain the uncertainty for the term. When we don't know how the term was estimated from the set of model realisations, further investigation is required in order to quantify its uncertainty.

\subsection{Literature available}

When there is no other data we can use, literature such as survey data, publications, handbooks and reports can be used for uncertainty quantification. We may follow the existing Australian or international standards for some specific individual terms. For example, recent study shows the uncertainty in the annual streamflows ranged from $\pm 4 \%$ to $\pm 41 \%$ of the reported flow in the Werrivee River catchment, Australia [Lowe et al., 2009].

A comprehensive literature review about uncertainty of water terms about Australian hydrological systems would be useful but has yet to be carried out. Adjustment, subject to further investigation, may be required to interpret and apply non-local literature, e.g., how to adjust according to the similarity between a catchment of interest and the one in the literature.

\subsection{Experts available}

For individual terms with little data and knowledge, domain experts or hydrologists may be available to provide domain judgement on uncertainty estimation. For example, experts may estimate the lower and upper 
bounds of uncertainty [Taylor and Kuyatt, 1994], which can then become very useful for estimating a Beta distribution uncertainty (Figure 1).

Once experts are identified, wherever possible, expert judgement regarding uncertainty should be elicited using an appropriate protocol such as Stanford/SRI protocol [Morgan et al., 1990; Penman et al., 2000]. Elicitation protocols can overcome the biases that may be introduced by the rules of thumb that experts use when formulating their judgements about uncertainty. The well-known expert elicitation protocol Stanford/SRI protocol consists of five steps: motivating, structuring, conditioning, encoding, and verification [Penman et al., 2000]. The elicited knowledge about uncertainty can be further integrated with observed data to infer uncertainty via Bayesian statistical techniques.

\subsection{Closing term}

This is a term that is back-calculated using water balance techniques. If there is only one term left without uncertainty estimation in a water account, we can use the uncertainty propagation law or LHS to estimate its uncertainty. The former is preferred only when all the other water terms have relatively small uncertainty and follow Gaussian distributions as discussed in Section 2.3.

\section{UNCERTAINTY AGGREGATION FOR HIERARCHICAL WATER ACCOUNTS}

Water accounts may form a hierarchical structure from different perspectives. For example, the proposed Australian water balance framework [Barratt and Nation, 2009] suggested three different hierarchies for water accounts from spatial, temporal and reporting details perspectives. The reporting entity may be at the sub-catchment, catchment, sub-national, and national levels. They naturally form a spatial hierarchy. At each level, a set of water accounts cover the whole nation without overlapping. Such exclusive coverage facilitates our uncertainty quantification from a lower spatial level. There are three levels for reporting details for one report entity: simple, intermediate and detailed water accounts. Under this framework, terms from detailed water accounts could be lumped to create intermediate and then simple water accounts.

If a term is aggregated from a subordinate level, we can directly aggregate uncertainty from that level provided that the uncertainty information related with that term is fully available at that level via the uncertainty propagation law or LHS. The former is again only preferred when all the component water terms have relatively small uncertainty and follow Gaussian distributions as discussed in Section 2.3.

\section{SOFTWARE IMPLEMENTATION}

Because most uncertainty analysis decisions will be made according to the decision tree, we mainly discuss the possible implementation mechanisms for the underlying uncertainty estimation techniques.

Most uncertainty analysis techniques could be implemented as functions or add-ins for spreadsheets such as Microsoft Excel or OpenOffice. Classic uncertainty analysis for measured data can be implemented via statistical functions in spreadsheets. Monte Carlo techniques can be implemented by add-ins like such as commercial software @Risk or Oracle Crystal Ball, or shareware MCSimNV. Bootstrapping and Latin Hypercube Sampling (LHS) could be done via bootstrap and LH-OAT, respectively.

Alternatively, these uncertainty analysis techniques can be carried out on standalone software such as Commercial software Analytica and freeware DAKOTA.

The uncertainty techniques suggested can also implemented in general statistical software such as R and SPlus. $\underline{\mathrm{R}}$ is a free software environment for statistical computing and graphics. It compiles and runs on a wide variety of UNIX platforms, Windows and MacOS, and can be downloaded at http://www.r-project.org/. $\underline{\mathrm{S}-}$ PLUS is commercial software, from which $\mathrm{R}$ is evolved.

\section{CONCLUSIONS AND DISCUSSIONS}

We presented a hierarchical decision-tree based uncertainty framework for assessing, quantifying and reporting quantitative uncertainty in water accounting. It provides a systematic guide to use practical techniques for quantifying uncertainty of line items in various water accounts, including the NWA. It can handle the hierarchical structure in water accounting and is not difficult to carry out.

The proposed framework, as a work in progress, is subject to further testing and refinement, especially after several case studies. There are several remaining issues (Figure 2). The framework leaves various decisions to be made by water accountants that are sometimes not straightforward. Some decision nodes may require refinement. For example, it may be also appropriate to distinguish models between known physical properties 
and models as approximations of reality. This framework may not quantify uncertainty for some quantities in water accounting. For example, without knowing the estimation method for a line item, we normally cannot derive its uncertainty range. This clearly requires further investigation. The framework will need some more sophisticated statistical techniques to holistically consolidate uncertainty within a single water account and spatio-temporal dependency among data. Another possible extension is to discuss quality assurance and reduction of uncertainty.

\section{ACKNOWLEDGMENTS}

This work is part of the water information research and development alliance between CSIRO's Water for a Healthy Country Flagship and the Bureau of Meteorology. The first author really appreciates Lisa Lowe for providing the data, marginal distribution functions and results for her work on the Werribee River catchment, Victoria, Australia. We appreciate the comments from Andrew Frost, Renée Kidson, Sri Srikanthan from the Bureau of Meteorology

\section{REFERENCES}

Barratt, D., and E. Nation (2009), A Proposed National Water Balance Framework: Discussion Paper, 1-42 pp, the Bureau of Meteorology.

Barratt, D., E. Nation, and A. Flanagan (2009), Water balance terms and their population using Water Act Regulations data, 1-45 pp.

Beven, K., and A. Binley (1992), The Future of Distributed Models - Model Calibration and Uncertainty Prediction, Hydrological Processes, 6(3), 279-298.

Bureau of Meteorology (2010), Pilot National Water Account, 1-359 pp, Commonwealth of Australia, Melbourne,http://www.bom.gov.au/water/nwa/document/Pilot_NWA_hi-res.pdf.

Davison, A. C., and D. V. Hinkley (1997), Bootstrap methods and their application, 582 pp., Cambridge University Press, Cambridge; New York, NY, USA.

Frost, A., C. Daamen, Steven Boxall, Grace Mitchell, Alison Oke, Catherine Beesley, Elisabetta Carrara, Derek Bacon, Eloise Nation, Sri Srikanthan, Champika Wethasinghe, Luke Shelley, and D. Barratt (2009), Methods Review: Water balance terms and their population using Water Act Regulations data $142 \mathrm{pp}$, Bureau of Meteorology, Melbourne.

Gupta, H. V., K. J. Beven, and T. Wagener (2005), Model Calibration and Uncertainty Estimation, in Encyclopedia of Hydrological Sciences, edited by M. Anderson, pp. 1-17, John Wiley \& Sons, Inc.

Jin, H., S. Barry, Y. G. Wang, and Q. Shao (2011), Systematically Reconciling Unbalanced and Uncertain Water Accounts, Water Resources Research, under review.

Kuczera, G., D. Kavetski, S. Franks, and M. Thyer (2006), Towards a Bayesian total error analysis of conceptual rainfall-runoff models: Characterising model error using storm-dependent parameters, Journal of Hydrology, 331(1-2), 161-177, DOI 10.1016/j.jhydrol.2006.05.010.

Lowe, L., T. Etchells, R. J. Nathan, B. Potter, and H. Malano (2006), Robust Water Accounting: What is it?, in 30th Hydrology and Water Resources Symposium: Past, Present \& Future, edited, pp. 382-389, Sandy Bay, Tasmania, Australia.

Lowe, L., T. Etchells, H. Malano, R. J. Nathan, and B. Potter (2009), Addressing uncertainties in water accounting, in 18th World IMACS/MODSIM Congress, edited, pp. 3626-3632, Cairns, Australia.

Morgan, M. G., M. Henrion, and M. Small (1990), Uncertainty: a guide to dealing with uncertainty in quantitative risk and policy analysis, 332 pp., Cambridge; New York.

Penman, J., I. E. Galbally, and Intergovernmental Panel on Climate Change. (2000), Good practice guidance and uncertainty management in national greenhouse gas inventories, Electronic book pp, IPCC National Greenhouse Gas Inventories Programme, Technical Support Unit, Kanagawa, Japan.

Shao, Q. X., A. van Dijk, S. Barry, and G. Turner (2009), Statistical challenges in water use accounting and data interpretation, 18th World Imacs Congress and Modsim09 International Congress on Modelling and Simulation, 3647-3653.

Taylor, B. N., and C. E. Kuyatt (1994), Guidelines for evaluating and expressing the uncertainty of NIST measurement results, 1994 ed., 1 v. pp., National Institute of Standards and Technology, Physics Laboratory, Gaithersburg, Md.

The Bureau of Meteorology (2010), Pilot National Water Account, 1-359 pp, Commonwealth of Australia, Melbourne,http://www.bom.gov.au/water/nwa/document/Pilot_NWA_hi-res.pdf.

Water Accounting Standards Board (2010), Exposure Draft of Australian Water Accounting Standard 1: Preparation and Presentation of General Purpose Water Accounting Reports, Commonwealth of Australia,http://www.bom.gov.au/water/standards/documents/ed_awas1_v1.0.pdf. 